\title{
STATIC DEFORMATIONS DUE TO THE FAULT SPREADING OVER SEVERAL LAYERS IN A MULTI-LAYERED MEDIUM PART I: DISPLACEMENT
}

\author{
Ryosuke SATo and Mitsuhiro MATSU'URA \\ Geophysical Institute, Faculty of Science, University of Tokyo, \\ Tokyo, Japan
}

(Received September 20, 1973)

\begin{abstract}
Surface deformations due to the fault spreading over several layers in a multi-layered medium are investigated. Machine program is developed by which contour map of vertical components and vector map of horizontal components around the focal area can be drawn, designating focal parameters as the inputs.

Some numerical examples of surface deformations are presented for the purpose of comparison with those obtained for a homogeneous semi-infinite model.

For a vertical dip-slip fault, for example, it is known that the vertical displacement at the surface is upheaval on one side of the strike of the fault and subsidence on the other side. For a multi-layered model, however, subsidence area appears even in the upheaval area mentioned above. We call this reverse area. Thus the displacement field shows different pattern from that for a semi-infinite model.
\end{abstract}

\section{Introduction}

Observed data on static deformation as well as on seismic waves enables us to determine focal parameters such as fault type, fault dimension, strike direction, dip angle, etc. In many studies, seismic wave data has been used mainly because of scarcity of good static field data around the focal area.

Recently, several investigators (SAVAGE and Hastie, 1969; HASTIE and and SAvage, 1970; Frtch and Scholz, 1971; Ando, 1971; CANitez and TokzOZz, 1972) determined focal mechanisms by use of the data on surface deformations, comparing with theoretical results obtained on the basis on an assumption of an isotropic homogeneous semi-infinite medium (CHINNERY, 1961; Maruyama, 1964; Press, 1965).

With accumulation of refined data, however, the assumption will be recognized to be too simple to explain the observations and theoretical investigation for a more realistic case is necessary, especially for great earthquakes 
whose faults are seemed to be spreaded within the crust.

Ben-Menahem and Gillon (1970) derived expressions of the static deformation when a point source is located in a layer overlying a semi-infinite medium. Sato (1971) developed the problem to the case of a multi-layered medium by matrix method and presented the numerical examples of the static deformations when the fault spreads in a layer overlying a semi-infinite medium. In his numerical examples, since he assumed a single layered crustal model, no definite difference from a semi-infinite model was found.

In the present paper, as the more realistic model, we consider the case when the fault spreads over several layers in a multi-layered medium and intend to complete the machine program to obtain surface deformations around the focal area due to the fault with any dip angle, any slip direction and any dimension.

\section{Analytical Representation of the Static Displacement at the Surface}

Derivation of analytical representations in the present paper is based on the formulation by SATo (1971). Details of the analytical formulation should

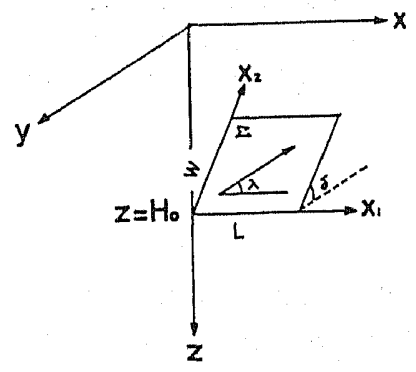

Fig. 1. Geometry of the dislocation model.

ô: dip angle,

$\lambda:$ slip angle,

$w$ : fault width,

$L:$ fault length,

$\Sigma:$ fault plane,

$H_{0}$ : focal depth,

$H$ : unit of length,

$d_{j}$ : thickness of the $j$-th layer,

$v_{p j}$ : velocity of dilatational wave in the $j$-th layer,

$v_{s j}$ : velocity of shear wave in the $j$-th layer.

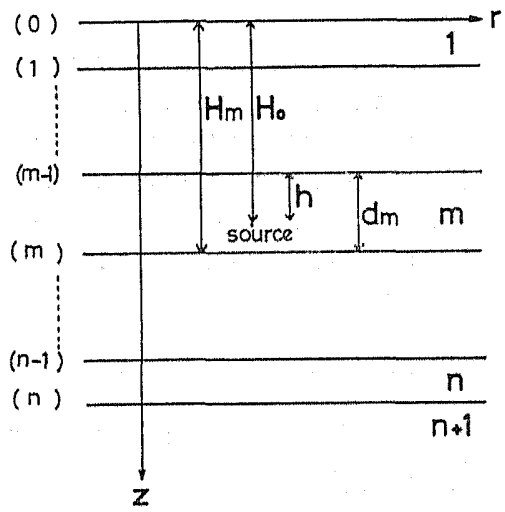

Fig. 2. Direction of axes and numbering of layers and interfaces.

$$
\begin{aligned}
H_{m} & =\sum_{j=1}^{m} d_{j}, \\
h & =H_{0}-H_{m-1}, \\
\beta j & =\xi d_{j}, \\
\beta & =\xi H . \\
r & =\xi h, \\
r & =\left\{\left(x-x_{1}^{2}\right)+\left(y+x_{2} \cos \delta\right)^{2}\right\}^{1 / 2}, \\
\varphi & =\tan ^{-1}\left\{\left(y+x_{2} \cos \delta\right) /\left(x-x_{1}\right)\right\}, \\
\alpha_{j} & =1-\left(v_{s j} / v_{p j}\right)^{2}, \\
\text { th layer, } &
\end{aligned}
$$


be referred to this paper, which is hereafter referred to as "paper I".

The displacement field at the surface due to the fault, which spreads over several layers in a multi-layered medium, is obtained by integrating point source solution over the fault plane. According to the "paper I", the solution for a point source, located in the $m$-th layer, is represented as in the following (geometries and notations are illustrated in Figs. 1 and 2):

$$
\boldsymbol{u}=\iint_{\Sigma} \boldsymbol{u}^{\prime} \mathrm{d} x_{1} \mathrm{~d} x_{2}
$$

where $\boldsymbol{u}^{\prime}$ is the solution for a point source and is given by

with

$$
\boldsymbol{u}^{\prime}=\left(u_{r}^{\prime}, u_{\varphi}^{\prime}, u_{z}^{\prime}\right) \text {, }
$$

$$
\begin{aligned}
& u_{r}^{\prime}=\frac{D}{4 \pi}\left\{\frac{\partial}{\partial r} \int_{0}^{\infty}\left[U_{r}\right]_{0} \mathrm{~d} \xi-\frac{1}{r} \frac{\partial}{\partial \varphi} \int_{0}^{\infty}\left[U_{\varphi}\right]_{0} \mathrm{~d} \xi\right\}, \\
& u_{\varphi}^{\prime}=\frac{D}{4 \pi}\left\{\frac{1}{r} \frac{\partial}{\partial \varphi} \int_{0}^{\infty}\left[U_{r}\right]_{0} \mathrm{~d} \xi+\frac{\partial}{\partial r} \int_{0}^{\infty}\left[U_{\varphi}\right]_{0} \mathrm{~d} \xi\right\}, \\
& u_{r}^{\prime}=\frac{D}{4 \pi} \int_{0}^{\infty} \xi\left[U_{z}\right]_{0} \mathrm{~d} \xi,
\end{aligned}
$$

and

$$
\begin{aligned}
{\left[U_{r(z)}\right]_{0}=} & \sum_{n=0}^{2}\left[\left\{(-1)^{n} U_{r(z), 1} \mathrm{e}^{-r}+U_{r(z), 2} \mathrm{e}^{-2 \beta_{m}+r}\right\} F_{n}\right. \\
& -\left\{(-1)^{n} U_{r(z), 3} \mathrm{e}^{-\gamma}+U_{r(8), 4} \mathrm{e}^{-2 \beta_{m}+r}\right\} G_{n} \\
& \left.+\alpha_{m}\left(\beta_{m}-\gamma\right)\left\{(-1)^{n} U_{r(z), 1} \mathrm{e}^{-r}-U_{r(z), 2} \mathrm{e}^{-2 \beta_{m}+r}\right\} G_{n}\right] \\
& \times J_{n}(\xi r) \cdot \exp \left(-\sum_{j=1}^{m-1} \beta_{j}\right), \\
{\left[U_{\xi}\right]_{0}=} & \sum_{n=1}^{2}\left[U_{\varphi, 1} \mathrm{e}^{-r}+(-1)^{n} U_{\varphi, 2} \mathrm{e}^{-2 \beta_{m}+r}\right] H_{n} J_{n}(\xi r) \exp \left(-\sum_{j=1}^{m-1} \beta_{j}\right) .
\end{aligned}
$$

Table 1.

\begin{tabular}{cccc}
\hline & \multicolumn{3}{c}{$n$} \\
\cline { 2 - 4 } & 0 & 1 & 2 \\
\hline$F_{n}$ & $\frac{1}{2}\left(2+a_{m}\right) a_{0}$ & $a_{1}$ & $\frac{1}{2}\left(2-\alpha_{m}\right) a_{2}$ \\
$G_{n}$ & $\frac{3}{2} a_{0}$ & $a_{1}$ & $\frac{1}{2} a_{2}$ \\
$H_{n}$ & - & $-a_{1}^{\prime}$ & $a_{2}^{\prime}$ \\
\hline
\end{tabular}

where

$$
\begin{array}{ll}
a_{0}=\sin \lambda \sin 2 \delta / 2, & \\
a_{1}=-\cos \lambda \cos \delta \cos \varphi+\sin \lambda \cos 2 \delta \sin \varphi, & a_{1}^{\prime}=\frac{\partial a_{1}}{\partial \varphi}, \\
a_{2}=\sin \lambda \sin 2 \delta \cos 2 \varphi / 2+\cos \lambda \sin \delta \sin 2 \varphi, & a_{2}^{\prime}=\frac{1}{2} \frac{\partial a_{2}}{\partial \varphi} .
\end{array}
$$


$U_{r(z), j}(j=1 \sim 4), U_{\varphi, j}(j=1,2)$ are expressed by Eqs. (48) and (75) in "paper I" and $F_{n}(n=0,1,2), G_{n}(n=0,1,2), H_{n}(n=1,2)$ by Eqs. (49) and (76), which are reproduced in Table 1.

\section{Evaluation of Integrals}

In order to carry out the integration, (3), with respect to $\xi, U_{r(z, \varphi), j}$ are approximated by analytical functions of the type $a+b \beta_{1}+c \mathrm{e}^{-\kappa \beta_{1}}$ in "paper I". When we treat the more realistic structural model of the crust, however, the functions of this form are not sufficient to fit the curve of $U_{r(z, \varphi), j}$, especially in the vicinity of $\beta_{1}=0$ (Fig. 3). We modify these analytical functions to the form, $a+b \beta_{1}+c \beta_{1}^{2}+d \mathrm{e}^{-x \beta_{1}}$. As is shown in Fig. 4, the results approximated by these analytical functions are very satisfactory. It is very important for the computation, whether these approximations are good or not, because the coefficients, $a, b, c, d$ and $\kappa$ contribute directly to the final results.

In "paper I", variable of integration was taken as $\beta_{1}\left(=\xi d_{1}\right)$, but when the fault spreads over several layeres, it is more useful to take the appropriate quantity, $H$, as unit of length, instead of $d_{1}$. With the transformation of the variable of integration from $\beta_{1}$ to $\beta(=\xi H)$, we redefine quantities such as $a$, $b, c, d, \kappa, s$ and $p$, as follows;

Corresponding to the representations in "paper I", we take

$$
\begin{array}{lll}
a \rightarrow a^{0}(=a), & b \rightarrow b^{0}\left\{=b\left(d_{1} / H\right)\right\}, & c \rightarrow c^{0}\left\{=c\left(d_{1} / H\right)^{2}\right\}, \\
d \rightarrow d^{0}(=d), & \kappa \rightarrow \kappa^{0}\left\{=\kappa\left(d_{1} / H\right)\right\}, &
\end{array}
$$

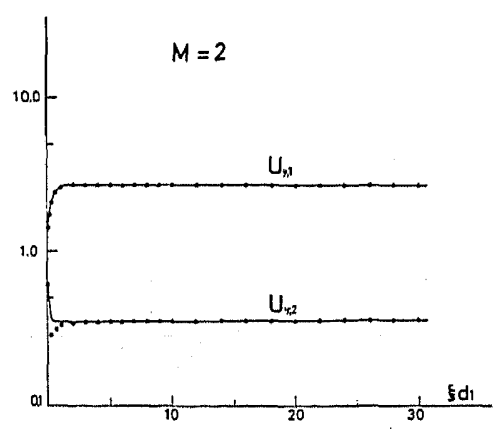

(a)

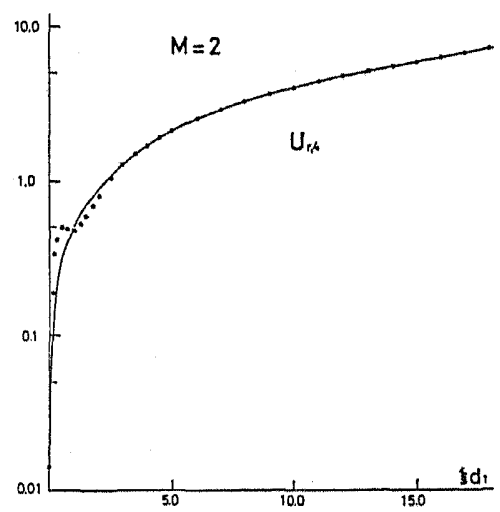

(b)

Fig. 3. Curves approximated by the function $a+b \beta_{1}+d \mathrm{e}^{-\kappa \beta_{1}}$ and values of $U_{r(z, \varphi), j}$, for the structural model I in Table 3 , as a function of $\beta_{1}=\xi d_{1}$. Approximate curves are shown by solid curves and computed values of $U_{r(x, \varphi), j}$ by dots. These illustrate examples of unsatisfactory approximations in the vicinity of $\beta_{1}=0$.

(a) Values of $U_{\varphi, 1}, U_{\varphi, 2}$ for the second layer of model I.

(b) Values of $U_{r, 4}$ for the second layer of model I. 


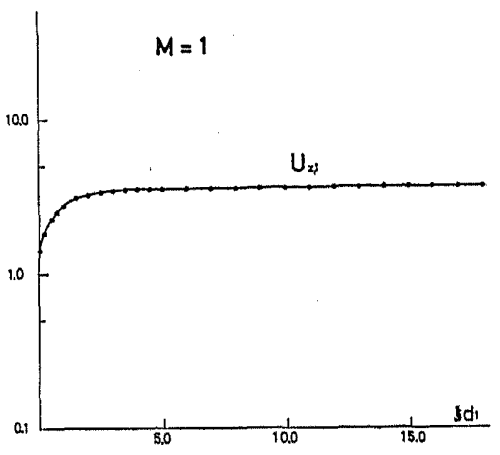

(a)

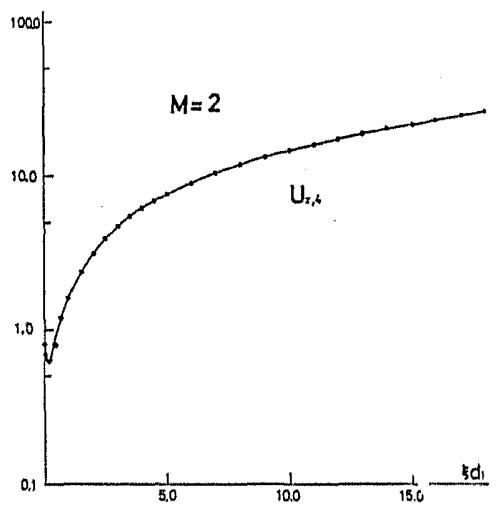

(c)

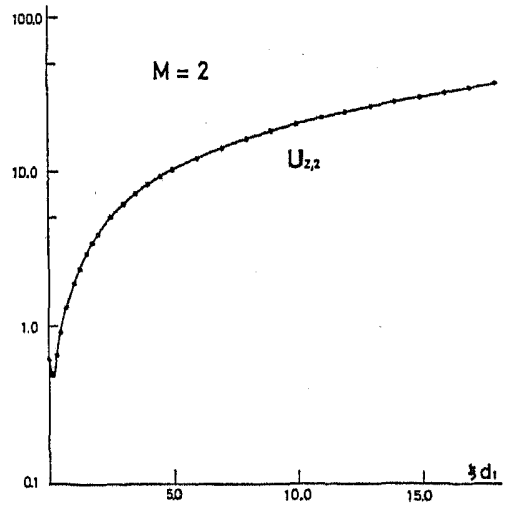

(b)

Fig. 4. Curves approximated by the function $a+b \beta_{1}+c \beta_{1}^{2}+d \mathrm{e}^{-\kappa \beta_{1}}$ (solid curves) and values of $U_{r(z, \varphi), j}$ (dots), for the structural model $\mathrm{I}$, as a function of $\beta_{1}=\xi d_{1}$. It is seen that approximation are satisfactory.

(a) Values of $U_{z, 1}$ for the first layer of model I.

(b) Values of $U_{x, 2}$ for the second layer of model I.

(c) Values of $U_{z, 4}$ for the second layer of model I.

$$
\begin{aligned}
a+b \beta_{1}+c \beta_{1}^{2}+d \mathrm{e}^{-\kappa \beta_{1}} & =a+b\left(\frac{d_{1}}{H}\right)(\xi H)+c\left(\frac{d_{1}}{H}\right)^{2}(\xi H)^{2}+d \mathrm{e}^{-\kappa\left(d_{1} / H\right)(\xi H)} \\
& =a^{0}+b^{0} \beta+c^{0} \beta^{2}+d^{0} \mathrm{e}^{-\kappa^{0} \beta},
\end{aligned}
$$

then,

$$
U_{r(z, \varphi), j} \approx a_{r(z, \varphi), j}^{0}+b_{r(z, \varphi), j}^{0} \beta+c_{r(z, \varphi), j}^{0} \beta^{2}+d_{r(x, \varphi), j}^{0} \mathrm{e}^{-x_{r(z, \varphi), j}^{0},}
$$

and

$$
\begin{array}{cll}
\text { unit of length } & d_{1} & \rightarrow H \text { (arbitrary), } \\
\text { variable of integration } & \beta_{1}\left(=\xi d_{1}\right) \rightarrow \beta(=\xi H), \\
s & r / d_{1} & \rightarrow r / H, \\
p & h / d_{1} & \rightarrow H H_{0} / H .
\end{array}
$$

Now following the process in "paper I", we can finally obtain expressions for displacements when the point source is located in the $m$-th layer as 


$$
\begin{aligned}
u_{r}^{\prime}= & \frac{D}{4 \pi H^{2}} \sum_{n=0}^{2}\left[\left\{(-1)^{n} \Lambda_{r, 1, n}^{(1)}(p)+\Lambda_{r, 2, n}^{(1)}\left(2 H_{m}-p\right)\right\} F_{n}\right. \\
& -\left\{(-1)^{n} \Lambda_{r, 3, n}^{(1)}(p)+\Lambda_{r, 4, n}^{(1)}\left(2 H_{m}-p\right)\right\} G_{n} \\
& \left.+\alpha_{m}\left(H_{m}-p\right)\left\{(-1)^{n} \Lambda_{r, 1, n}^{(2)}(p)-\Lambda_{r, 2, n}^{(2)}\left(2 H_{m}-p\right)\right\} G_{n}\right] \\
& -\frac{D}{4 \pi H^{2}} \sum_{n=1}^{2}\left[\left\{\Lambda_{\varphi, 1, n}^{(3)}(p)+(-1)^{n} \Lambda_{\varphi, 2, n}^{(3)}\left(2 H_{m}-p\right)\right\} \frac{\mathrm{d} H_{n}}{\mathrm{~d} \varphi}\right] \\
u_{\varphi}^{\prime}= & \frac{D}{4 \pi H^{2}} \sum_{n=1}^{2}\left[\left\{(-1)^{n} \Lambda_{r, 1, n}^{(3)}(p)+\Lambda_{r, 2, n}^{(3)}\left(2 H_{m}-p\right)\right\} \frac{\mathrm{d} F_{n}}{\mathrm{~d} \varphi}\right. \\
& -\left\{(-1)^{n} \Lambda_{r, 3, n}^{(3)}(p)+\Lambda_{r, 4, n}^{(3)}\left(2 H_{m}-p\right)\right\} \frac{\mathrm{d} G_{n}}{\mathrm{~d} \varphi} \\
& \left.+\alpha_{m}\left(H_{m}-p\right)\left\{(-1)^{n} \Lambda_{r, 1, n}^{(4)}(p)-\Lambda_{r, 2, n}^{(4)}\left(2 H_{m}-p\right)\right\} \frac{\mathrm{d} G_{n}}{\mathrm{~d} \varphi}\right] \\
& +\frac{D}{4 \pi H^{2}} \sum_{n=1}^{2}\left[\left\{\Lambda_{\varphi, 1, n}^{(1)}(p)+(-1)^{n} \Lambda_{\varphi, 2, n}^{(1)}\left(2 H_{m}-p\right)\right\} H_{n}\right], \\
& D \\
u_{z}^{\prime}= & \frac{D}{4 \pi H^{2}} \sum_{n=0}^{2}\left[\left\{(-1)^{n} \Lambda_{z, 1, n}^{(5)}(p)+\Lambda_{z, 2, n}^{(5)}\left(2 H_{m}-p\right)\right\} F_{n}\right. \\
& -\left\{(-1)^{n} \Lambda_{z, 3, n}^{(5)}(p)+\Lambda_{z, 4, n}^{(5)}\left(2 H_{m}-p\right)\right\} G_{n} \\
& \left.+\alpha_{m}\left(H_{m}-p\right)\left\{(-1)^{n} \Lambda_{z, 1, n}^{(6)}(p)-\Lambda_{z, 2, n}^{(6)}\left(2 H_{m}-p\right)\right\} G_{n}\right],
\end{aligned}
$$

with

$$
\begin{aligned}
\frac{1}{H^{2}} \Lambda_{r(\varphi), j, n}^{(1)}(p) \equiv & \frac{1}{2 H^{2}}\left[a_{r(\varphi), j}^{0}\left\{I_{1, n-1}(p)-I_{1, n+1}(p)\right\}\right. \\
& +b_{r(\varphi), j}^{0}\left\{I_{2, n-1}(p)-I_{2, n+1}(p)\right\}+c_{r(\varphi), j}^{0}\left\{I_{3, n-1}(p)-I_{3, n+1}(p)\right\} \\
& \left.+d_{r(\varphi), j}^{0}\left\{I_{1, n-1}(\kappa+p)-I_{1, n+1}(\kappa+p)\right\}\right] \\
\frac{1}{H^{2}} \Lambda_{r(\varphi), j, n}^{(2)}(p) \equiv & \frac{1}{2 H^{2}}\left[a_{r(\varphi), j}^{0}\left\{I_{2, n-1}(p)-I_{2, n+1}(p)\right\}\right. \\
& +b_{r(\varphi), j}^{0}\left\{I_{3, n-1}(p)-I_{3, n+1}(p)\right\}+c_{r(\varphi), j}^{0}\left\{I_{4, n-1}(p)-I_{4, n+1}(p)\right\} \\
& \left.+d_{r(\varphi), j}^{0}\left\{I_{2, n-1}(\kappa+p)-I_{2, n+1}(\kappa+p)\right\}\right], \\
\frac{1}{H^{2}} \Lambda_{r(\varphi), j, n}^{(3)}(p) \equiv & \frac{1}{2 n H^{2}}\left[a_{r(\varphi), j}^{0}\left\{I_{1, n-1}(p)+I_{1, n+1}(p)\right\}\right. \\
& +b_{r(\varphi), j}^{0}\left\{I_{2, n-1}(p)+I_{2, n+1}(p)\right\}+c_{r(\varphi), j}^{0}\left\{I_{3, n-1}(p)+I_{3, n+1}(p)\right\} \\
& \left.+d_{r(\varphi), j}^{0}\left\{I_{1, n-1}(\kappa+p)+I_{1, n+1}(\kappa+p)\right\}\right], \\
\frac{1}{H^{2}} \Lambda_{r(\varphi), j, n}^{(4)}(p) \equiv & \frac{1}{2 n H^{2}}\left[a_{r(\varphi), j}^{0}\left\{I_{2, n-1}(p)+I_{2, n+1}(p)\right\}\right. \\
& +b_{r(\varphi), j}^{0}\left\{I_{3, n-1}(p)+I_{3, n+1}(p)\right\}+c_{r(\varphi), j}^{0}\left\{I_{4, n-1}(p)+I_{4, n+1}(p)\right\} \\
& \left.+d_{r(\varphi), j}^{0}\left\{I_{2, n-1}(\kappa+p)+I_{2, n+1}(\kappa+p)\right\}\right],
\end{aligned}
$$




$$
\begin{aligned}
& \frac{1}{H^{2}} \Lambda_{z, j, n}^{(\delta)}(p) \equiv \frac{1}{H^{2}}\left[a_{z, j}^{0} I_{1, n}(p)+b_{z, j}^{0} I_{2, n}(p)+c_{z, j}^{0} I_{3, n}(p)+d_{z, j}^{0} I_{1, n}(\kappa+p)\right], \\
& \frac{1}{H^{2}} \Lambda_{z, j, n}^{(\theta)}(p) \equiv \frac{1}{H^{2}}\left[a_{z, j}^{0} I_{2, n}(p)+b_{z, j}^{0} I_{3, n}(p)+c_{z, j}^{0} I_{4, n}(p)+d_{z, j}^{0} I_{1, n}(\kappa+p)\right],
\end{aligned}
$$

where

$$
I_{m, n}(x)=\int_{0}^{\infty} \beta^{m} \mathrm{e}^{-x \beta} J_{n}(s \beta) \mathrm{d} \beta
$$

whose values with necessary combinations of $m$ and $n$ are tabulated in Table 2 .

Table 2. $\quad I_{m, n}=\int_{0}^{\infty} \beta^{m} \mathrm{e}^{-x \beta} J_{n}(s \beta) \mathrm{d} \beta$

\begin{tabular}{ccccc}
\hline & \multicolumn{4}{c}{$n$} \\
\cline { 2 - 5 } & 0 & 1 & 2 & 3 \\
\hline 1 & $\frac{x}{R^{3}}$ & $\frac{s}{R^{3}}$ & $\frac{s^{2}(2 R+x)}{R^{3}(R+x)^{2}}$ & $\frac{s^{3}(3 R+x)}{R^{3}(R+x)^{3}}$ \\
2 & $\frac{2 x^{2}-s^{2}}{R^{5}}$ & $\frac{3 s x}{R^{5}}$ & $\frac{3 s^{2}}{R^{5}}$ & $\frac{s^{3}\left(8 R^{2}+9 R x+3 x^{2}\right)}{R^{5}(R+x)^{3}}$ \\
3 & $\frac{3 x\left(2 x^{2}-3 s^{2}\right)}{R^{7}}$ & $\frac{3 s\left(4 x^{2}-s^{2}\right)}{R^{7}}$ & $\frac{15 s^{2} x}{R^{7}}$ & $\frac{15 s^{3}}{R^{3}}$ \\
4 & $\frac{3\left(8 x^{2}\left(x^{2}-3 s^{2}\right)+3 s^{4}\right)}{R^{9}}$ & $\frac{15 s x\left(4 x^{2}-3 s^{2}\right)}{R^{9}}$ & $\frac{15 s^{2}\left(6 x^{2}-s^{2}\right)}{R^{9}}$ & $\frac{105 s^{3} x}{R^{9}}$ \\
\hline & $* R=\sqrt{x^{2}+s^{2}}$ & & &
\end{tabular}

The surface displacements due to the fault with length $L$ and width $w$ can be found by replacing $x, y, H_{0}$ in the above expressions by $x-x_{1}, y+x_{2} \cos \delta$, $H_{0}-x_{2} \sin \delta$, respectively (or $r, \cos \varphi, \sin \varphi$ by $r^{\prime}=\sqrt{\left(x-x_{1}\right)^{2}+\left(y+x_{2} \cos \delta\right)^{2}}$, $\left.\left(x-x_{1}\right) / r^{\prime},\left(y+x_{2} \cos \delta\right) / r^{\prime}\right)$, and integrating from 0 to $L$ with respect to $x_{1}$ and from 0 to $w$ with respect to $x_{2}$.

Thus, denoting

$$
x_{1} / H=\eta, \quad x_{2} / H=\zeta,
$$

we have

$$
\boldsymbol{u}=\int_{0}^{L} \mathrm{~d} x_{1} \int_{0}^{w} \mathrm{~d} x_{2} \boldsymbol{u}^{\prime}\left(x_{1}, x_{2}\right)=H^{2} \int_{0}^{L / H} \int_{0}^{w / H} \boldsymbol{u}^{\prime}(\eta, \zeta) \mathrm{d} \eta \mathrm{d} \zeta
$$

Numerical integration in the present paper is carried out by using GaussLobatto's quadrature formula in $x_{2}$-direction and trapezoidal rule in $x_{1}$-direction (Fig. 1). 


\section{Program System}

\subsection{Explanation of programs}

Main purpose of the present paper is to establish a machine computation routine system to obtain the static deformation by putting the fault parameters into the computer.

The program system consists of five parts. Block diagram is shown in the following chart:

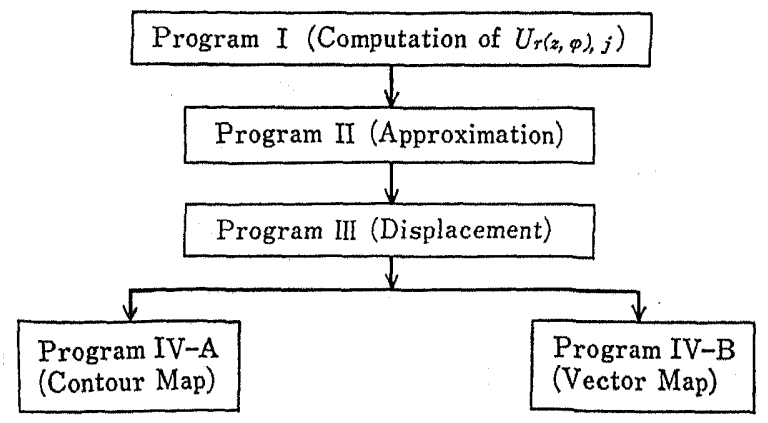

(a) Program I: Calculation of $U_{r(z, \varphi), j}$

$U_{r(z, \varphi), j}$ are calculated as a function of $\beta_{1}\left(=\xi d_{1}\right)$, for the given structure and given layer number, $M$, where the source is located ( $M$ is the same as $m$ in Fig. 2).

$U_{r(z, \varphi), j}$ are defined by Eqs. (45) and (75) in "paper I".

(b) Program II: Least squares approximation

In this program, we approximate $U_{r(x, \varphi), j}$, which were calculated in the Program I, by the analytical functions, $a+b \beta_{1}+c \beta_{1}^{2}+d \mathrm{e}^{-\kappa \beta_{1}}$, using the least squares method for non-linear system (SCABROUGH, 1955).

For given initial guesses, which are appropriately given in the program, the least squares method is repeated successively thirty times. If the difference of values of sum of squares of residuals (s.s.r.) between one step and the next step, divided by the sum of squares of $U_{r(z, \varphi), j}$ values in the range of $\beta_{1}$ under consideration $\left(=\sum_{i}\left\{U_{r,(z, \varphi), j}\left(\beta_{1 i}\right)\right\}^{2}\right)$, become smaller than $10^{-5}$, we adopt the values of $a_{r(z, \varphi), j}, b_{r(z, \varphi), j}, c_{r(z, \varphi), j}, d_{r(z, \varphi), j}$ and $\kappa_{r(z, \varphi), j}$ at this step, except for the case when the value of s.s.r. divided by $\sum_{i}\left\{U_{r(z, \varphi), j}\left(\beta_{1 i}\right)\right\}^{2}$ is greater than $10^{2}$. If the approximation does not converge for the first initial guesses, the program takes another initial guesses and the same process is repeated. If the approximation still does not converge for three different initial guesses, we adopt the values which give the least s.s.r. value in $90(30 \times 3)$ steps. However, there was no such a case in our experience. 
Computed results of Program I and II depend on the given crustal structure and independent of fault parameters. Hence when the structure is fixed, out put of Program II, or values of $a, b, c, d$ and $\kappa$, can be used for any types of faults in this structure.

(c) Program III (Displacement)

Using the output of Program II, the final displacement is obtained by Program III. The displacement field can be obtained for the fault with any dip-angle, any slip-direction and any dimension. If both the fault length and the fault width are designated as zero, then outputs give surface displacements due to a point source. If they are not zero, the numerical integration must be carried out over the fault plane. Two dimensional integration, however, usually takes rather long computational time even when obtaining one value at the surface and hence in the present paper the following method is adopted: The two dimensional rectangular fault can be regarded as supperposition of many line sources in numerical sense. First the displacement field over the surface due to a line source along $x_{2}$-axis (Fig. 1) is computed by using the Gauss-Lobatto's quadrature formula. The displacement field due to the neighboring line source apart from the first one by $\Delta x_{1}$ along $x_{1}$-direction can be obtained by sliding the first displacement field by $\Delta x_{1}$ on the surface, along $x$-direction. Sum of these displacements gives total fields due to the two dimensional fault of $w \times \Delta x_{1}$. Repeating this process until when $n \Delta x_{1}$ becomes $L$, we get total displacement fields due to the fault of $w \times L$. This implies the application of trapezoidal rule along $x_{1}$-direction. In this sence, $\Delta x_{1}$ is the interval of subdivision of numerical integration and is also the interval of mesh points at the surface where displacements should be calculated, and hence it seems to be necessary to take $\Delta x_{1}$ as small as possible which again requires long computational time. However, application of interpolation formula is feasible to obtain surface displacements with sufficiently dense mesh points.

According to this method, computation of displacement field due to only one line source is needed even for the two dimensional rectangular fault and computational time is considerably saved.

As is suggested by expressions for displacement field in an infinite medium, contributions from four corners of the fault are important. This is one of reasons why the Gauss-Lobatto's quadrature formula is used when calculating surface displacements due to the line source.

Distribution of the dislocation over the fault plane are given by Statement Function in Program III.

By designating appropriate unit length, the displacement field with necessary area can be found.

(d) Program IV-A: Contour map of the vertical displacement 
By putting the output card deck of the Program III into this program, we get the contour map of the vertical component of displacement.

(e) Program IV-B: Vector map of the horizontal displacement

By putting the output data of Program III $\left(U_{x}\right.$ and $\left.U_{y}\right)$ into this program, the vector map of the horizontal component $\left(U_{H}=\sqrt{U_{x}^{2}+U_{y}^{2}}\right)$ is obtained.

Final results by this program system (contour map and vector map) are presented in Figs. 5 and 6.

DISPLACEMENT VERTICAL COMPONENT
FOCAL DEPTH $=5.0 \mathrm{KM}$ UNIT LENGTH $=10.0 \mathrm{KM}$ DIP ANGLE $=60^{\circ}$ SLIP ANGLE $=-90^{\circ}$

SEMI-INFINITE MODEL POINT SOURCE

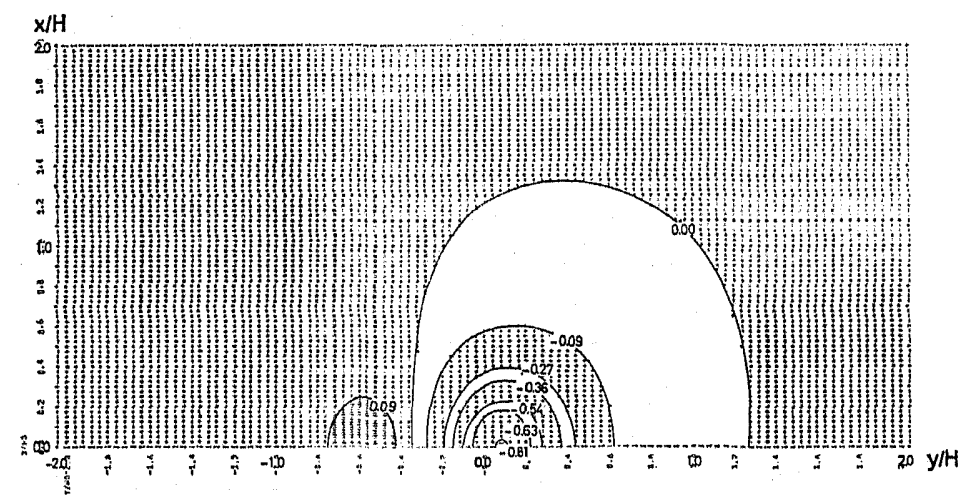

Fig. 5. Contour map of vertical displacements due to the dip-slip fault, drawn by Program IV-A.

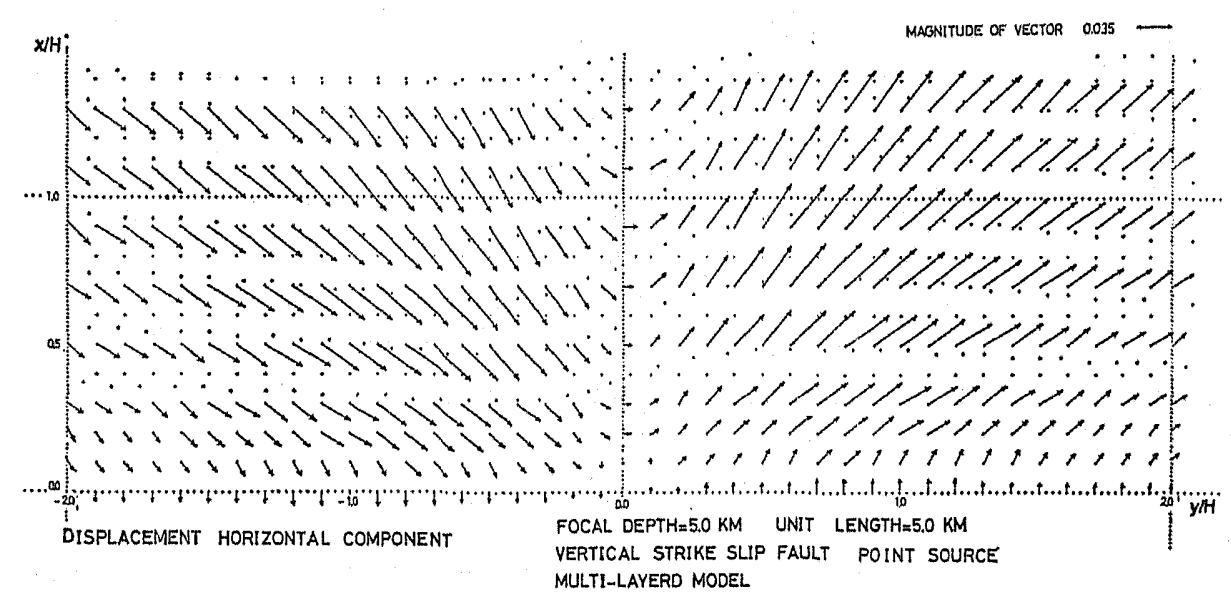

Fig. 6. Vector map of horizontal displacements due to the vertical strike-slip fault, plotted by Program IV-B. 


\subsection{Flow chart}

The flow charts of each part of the program system are shown in the following:

\section{Program I}

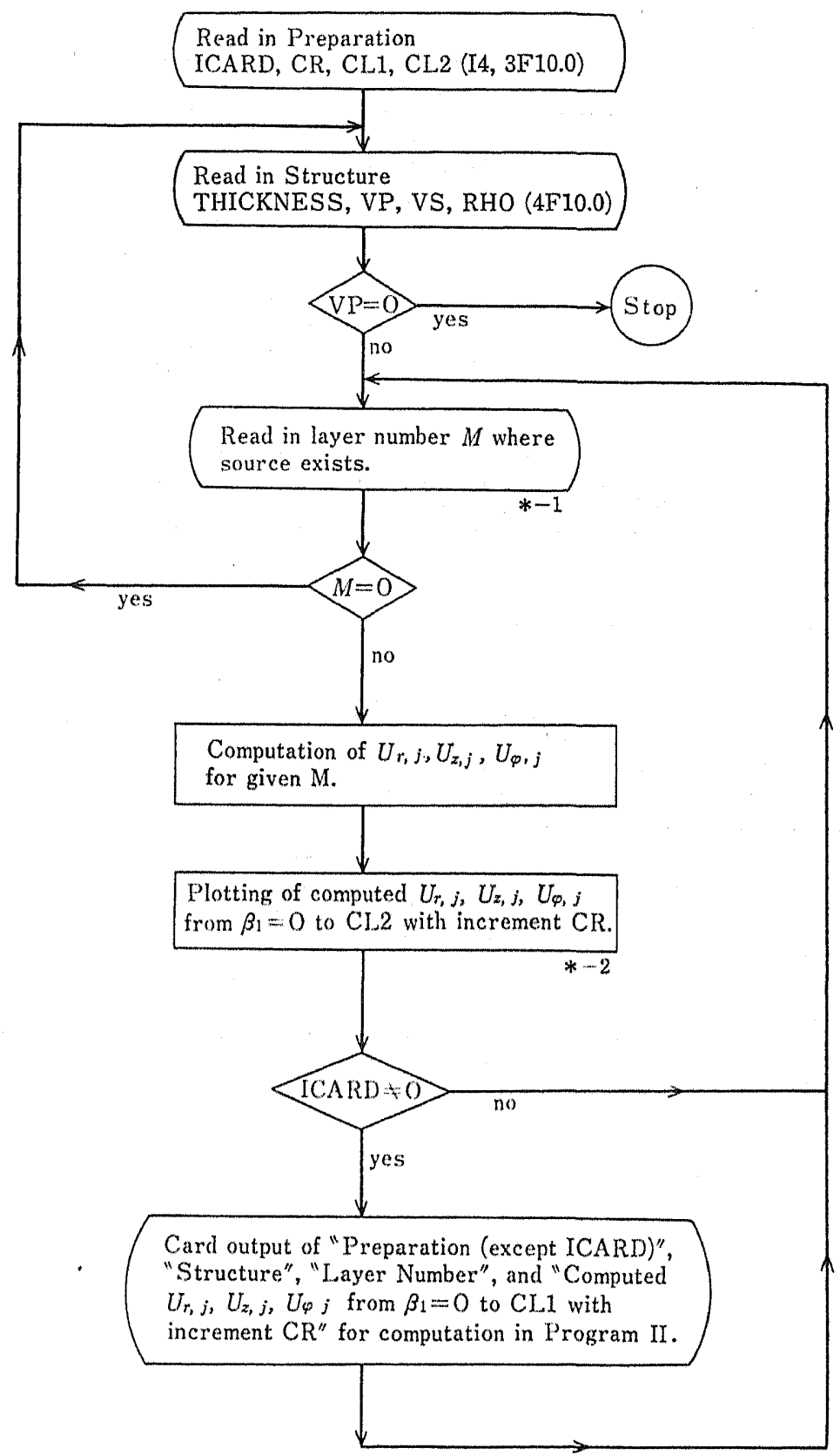




\section{Program II. (Approximation)}

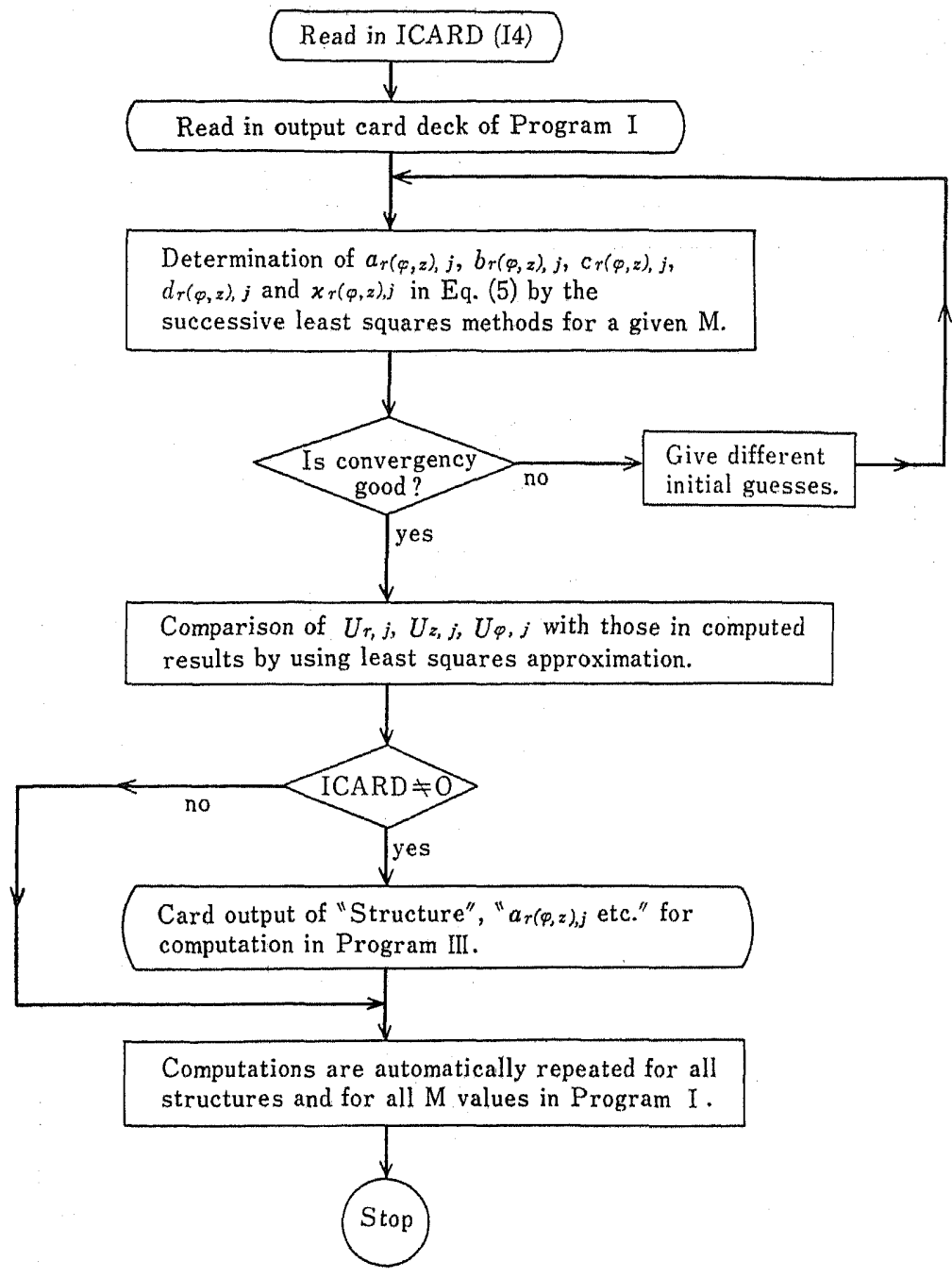




\section{Program III (Displacement)}

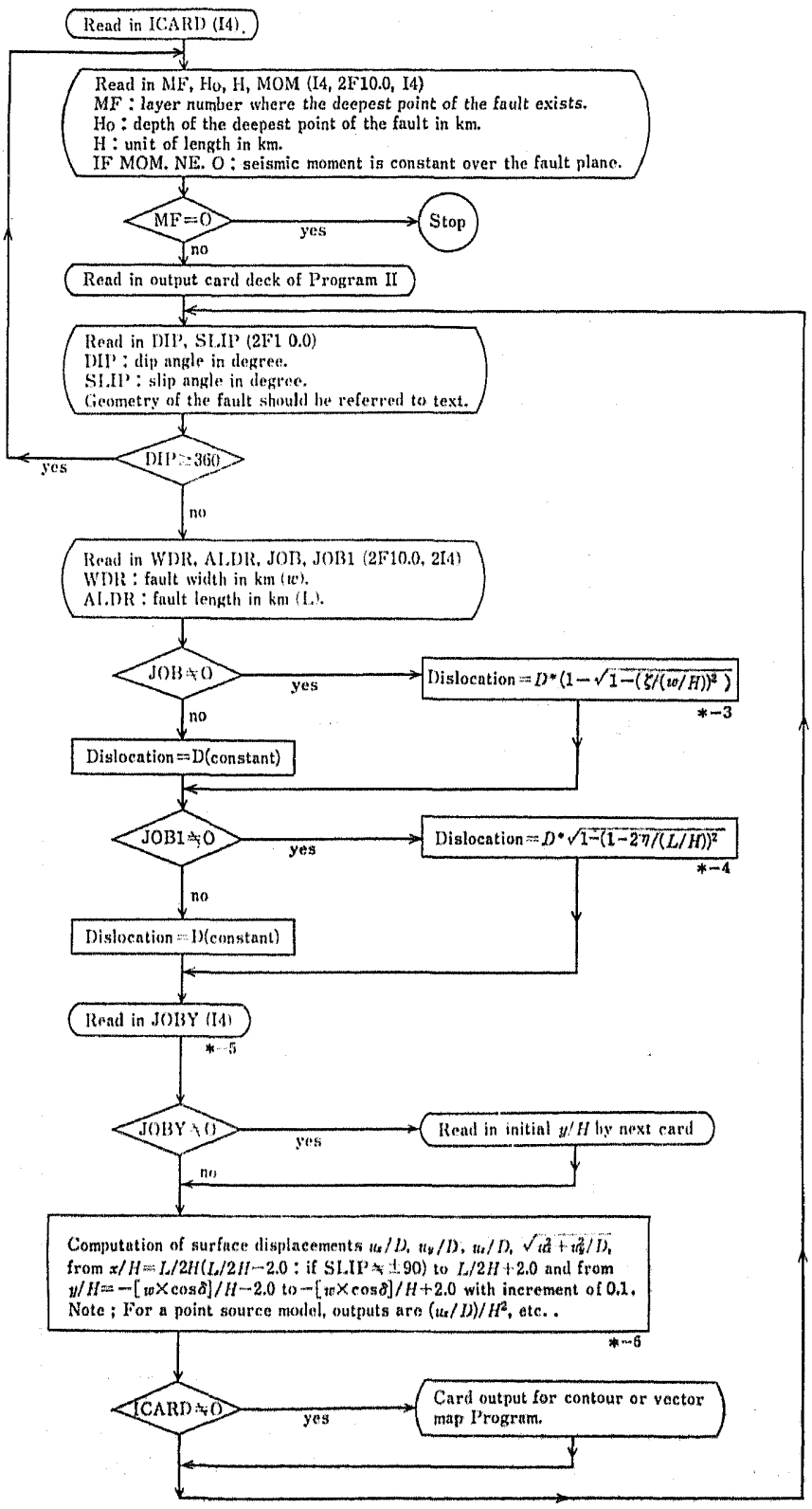




\section{Program IV-A (Contour Map)}

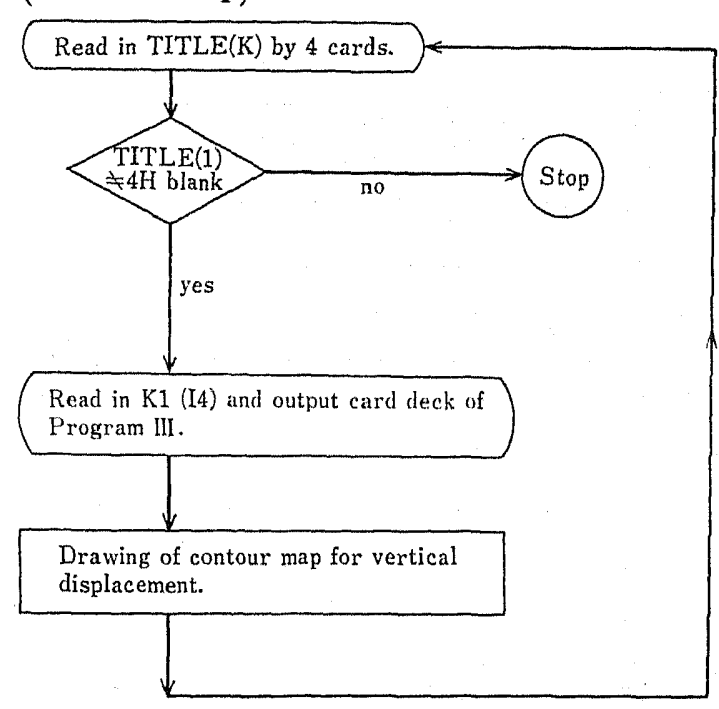

\section{Program IV-B (Vector Map)}

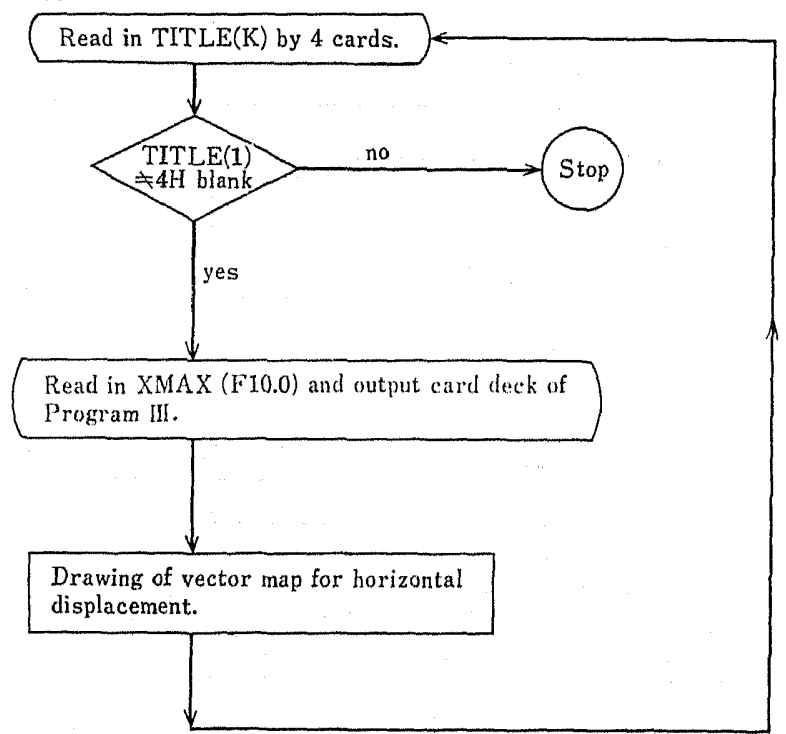

*-1: $\quad M$ is the layer number the where the source exists. If the fault spread over three layers, say, first, second third, then $M S$ are read by three cards and a blank card is put to go to the other structure-read-in. In the case of a semi-infinite model, $M$ is taken as 1 .

*-2: By knowing rough behaviors of $U_{r, j}, U_{z, j}, U_{\varphi, j}$ appropriateness of approximation method in Program II can be judged.

*-3: Distribution-function of the dislocation for the $x_{2}$-direction.

*-4: Distribution-function of the dislocation for the $x_{1}$-direction.

*-5: When the calculation is not completed within one job because of time limit, starting values of the next job is given by this information.

*-6: If the slip-angle is $90^{\circ}$ or $-90^{\circ}$, the displacement field at the surface is symmetry as to $x=\mathrm{ALD} / 2$, and so, in this case, the displacement field in the region of $x \geqq A L D / 2$ is computed. 


\section{Numerical Examples}

Several numerical examples are presented in this section by using machine program system developed in this study. In order to compare surface displacements for a multi-layered medium with those for a semi-infinite medium, we consider here two models, model I and II, which are tabulated in Table 3. Modle II is the averaged structure of model I by

$$
\begin{gathered}
\frac{1}{v_{p}}=\frac{1}{\sum_{i} d_{i}} \sum_{i}\left(\frac{d_{i}}{v_{p i}}\right), \\
\frac{1}{v_{s}}=\frac{1}{\sum_{i} d_{i}} \sum_{i}\left(\frac{d_{i}}{v_{s i}}\right), \\
\rho=\frac{1}{\sum_{i} d_{i}} \sum_{i}\left(d_{i} \rho_{i}\right) .
\end{gathered}
$$

i) Focal depth variation (Figs. 7 and 8)

The magnitude of surface deformations for model I along $y$-axis (Fig. 1) is compared with that for model II for various focal depths. Unit length is taken as $10 \mathrm{~km}$. Since this is the point sourca case, unit of displacement is $D / H^{3}$.

Comparing the horizontal component for model I with that for model II in the case of the vertical strike-slip fault (Fig. 7), it is found that the maximum deformation for model I is rather greater than that for model II, but the difference in the pattern of the displacement between two models is not so obvious.

In Fig. 8, the vertical components of the displacements are shown for the vertical dip-slip fault. In this case, remarkable difference between them is obtained. For the case of model II (Fig. 8(b)), the displacement field shows

Table 3.

model I

\begin{tabular}{ccccc}
\hline layer number & thickness $(\mathrm{km})$ & $V_{\mathrm{p}}(\mathrm{km} / \mathrm{sec})$ & $V_{\mathrm{s}}(\mathrm{km} / \mathrm{sec})$ & density $\left(\mathrm{gr} / \mathrm{cm}^{3}\right)$ \\
\hline 1 & 1.70 & 3.56 & 2.37 & 2.00 \\
2 & 2.80 & 5.50 & 3.10 & 2.50 \\
3 & 10.60 & 6.15 & 3.40 & 2.70 \\
4 & 16.90 & 6.70 & 3.70 & 2.88 \\
5 & $\infty$ & 8.00 & 4.50 & 3.28 \\
\hline
\end{tabular}

model II

\begin{tabular}{ccccc}
\hline layer number & thickness $(\mathrm{km})$ & $V_{\mathrm{p}}(\mathrm{km} / \mathrm{sec})$ & $V_{\mathrm{s}}(\mathrm{km} / \mathrm{sec})$ & density $\left(\mathrm{gr} / \mathrm{cm}^{3}\right)$ \\
\hline 1 & $\infty$ & 6.12 & 3.44 & 2.74 \\
\hline
\end{tabular}




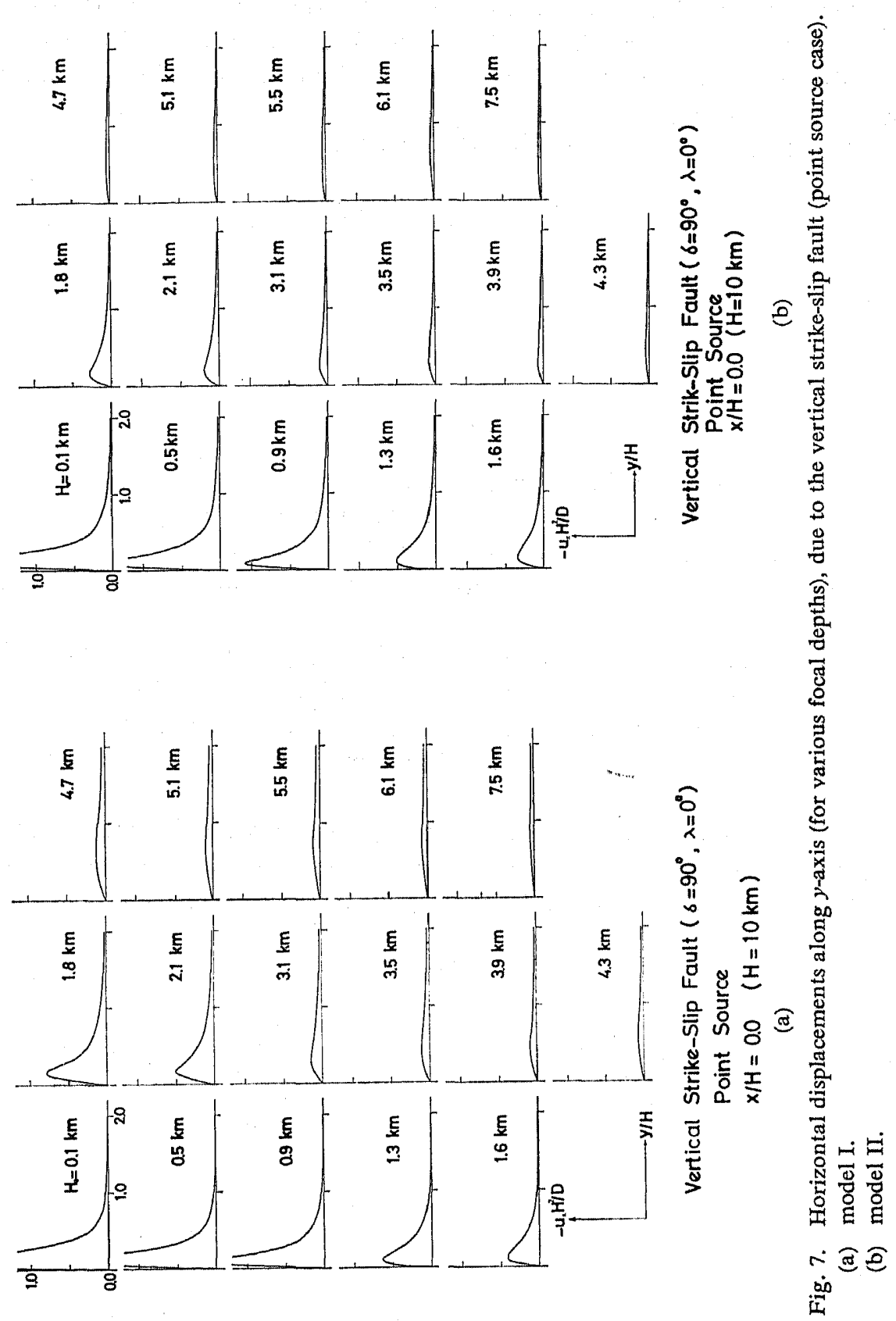



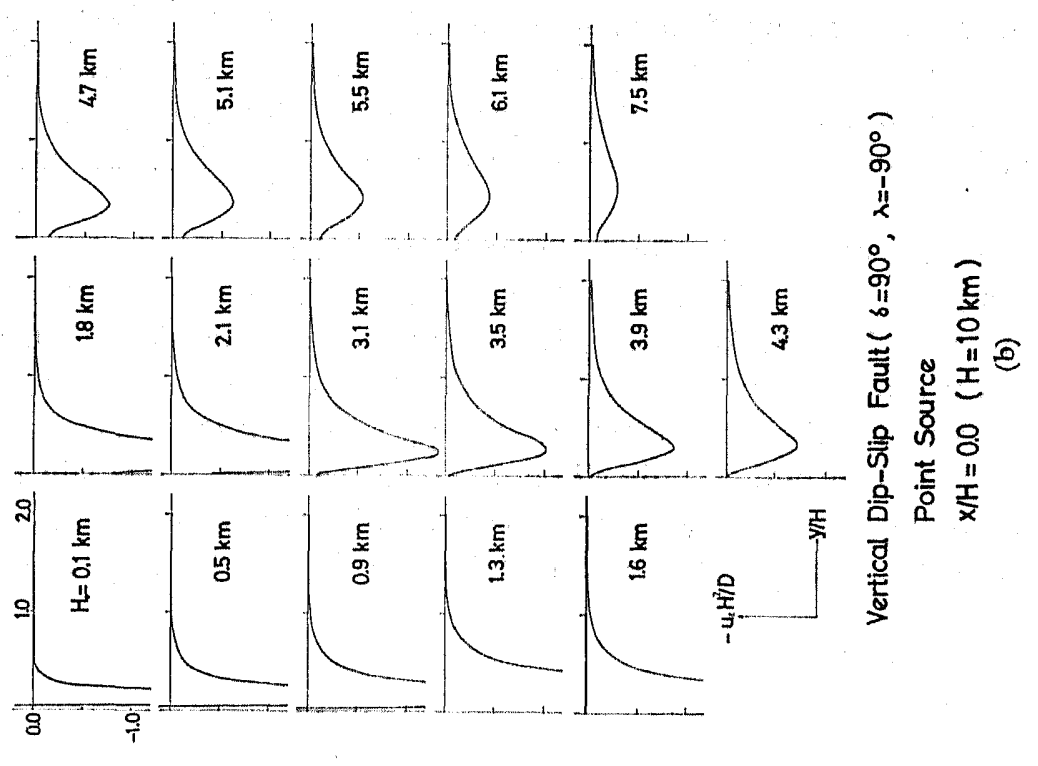

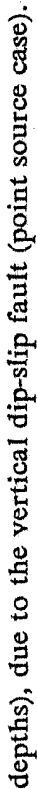
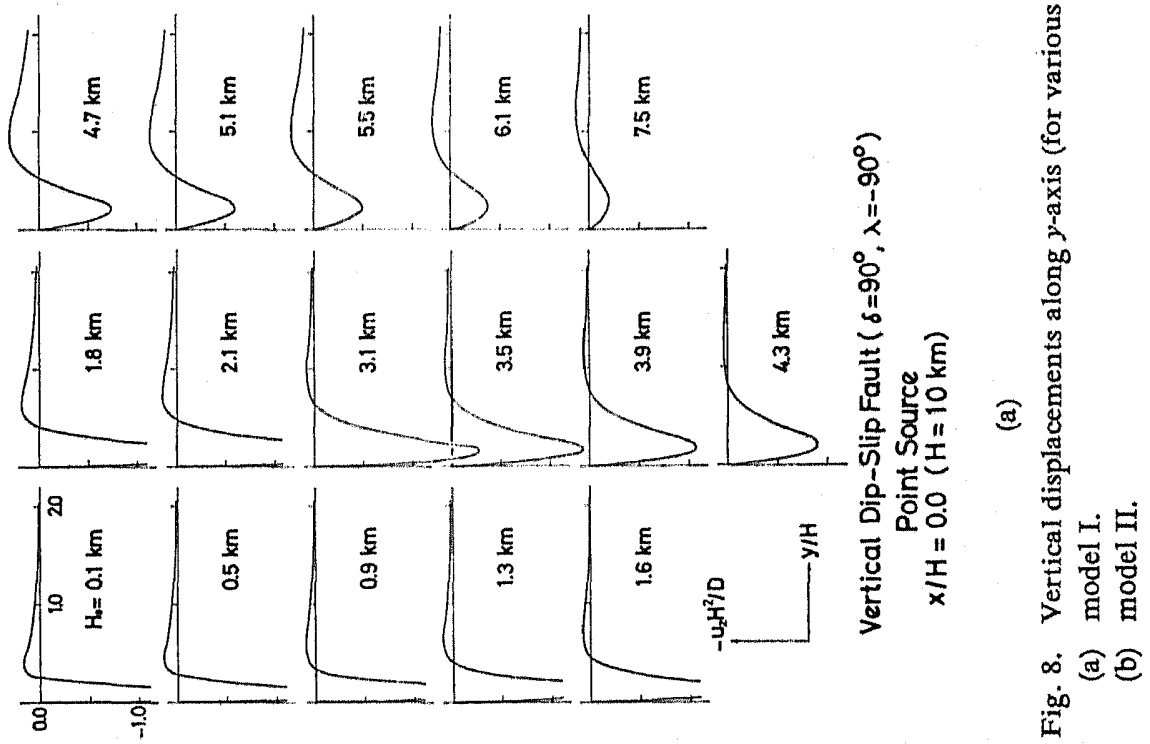
large subsidence in the neighbourhood of $y=0$, and then approaches to the zero value monotonously. On the other hand, for the case of model I, the obvious uplift region appears, following the subsidence region in the neighbourhood of the source. This is clearly seen in Fig. 8(a) when $H_{0}$ is greater than $4.5 \mathrm{~km}$, or the source is in the third layer. We call this region, where the direction of the deformation is opposite to that for the semi-infinite model, reverse area.

ii) The case of the point source (Figs. 9, 10 and 11)

The patterns of the displacement field at the surface are compared for several fault models. The focal depth is taken as $5 \mathrm{~km}$ and unit length $10 \mathrm{~km}$.

When drawing the contour map of the vertical component for model I and comparing with that for model II, the appearance of the reverse area is more clearly seen for both the dip-slip fault (Fig. 9) and the strike-slip fault (Fig. 10), although vertical displacements are smaller for the latter fault. As is expected from these figures, the pattern for the tilt will be very different

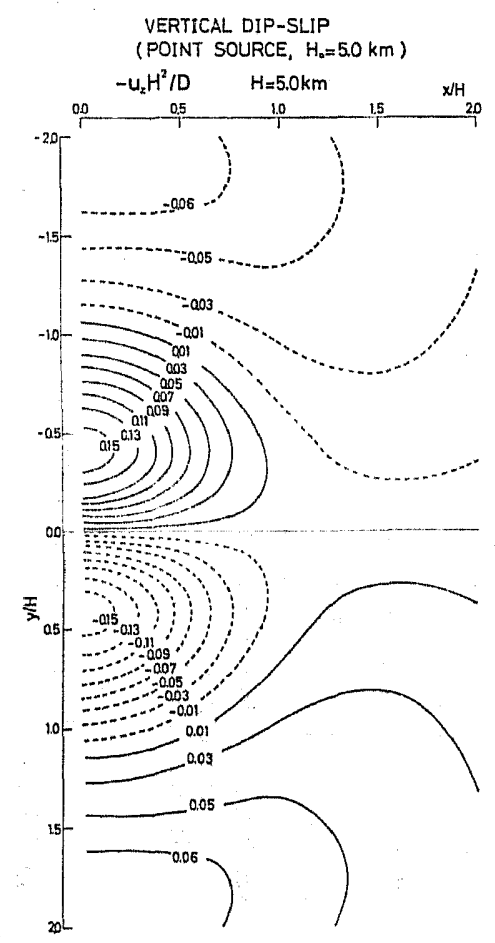

(a)

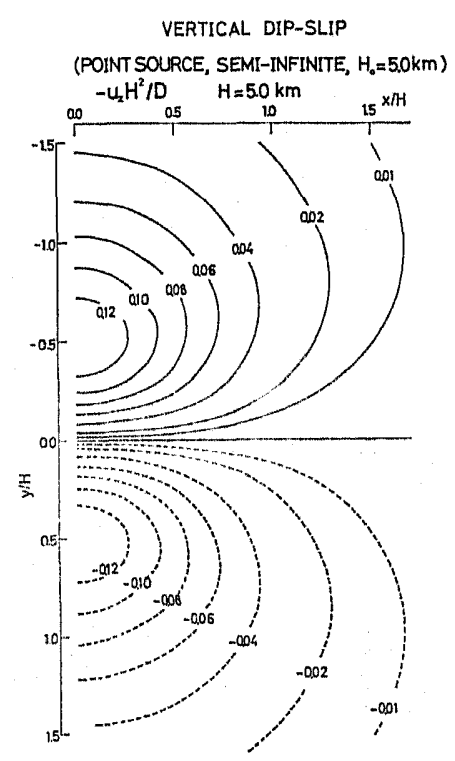

(b)

Fig. 9. Contour map of vertical displacements at the surface due to the vertical dipslip fault (point source case).

(a) model I.

(b) model II. 


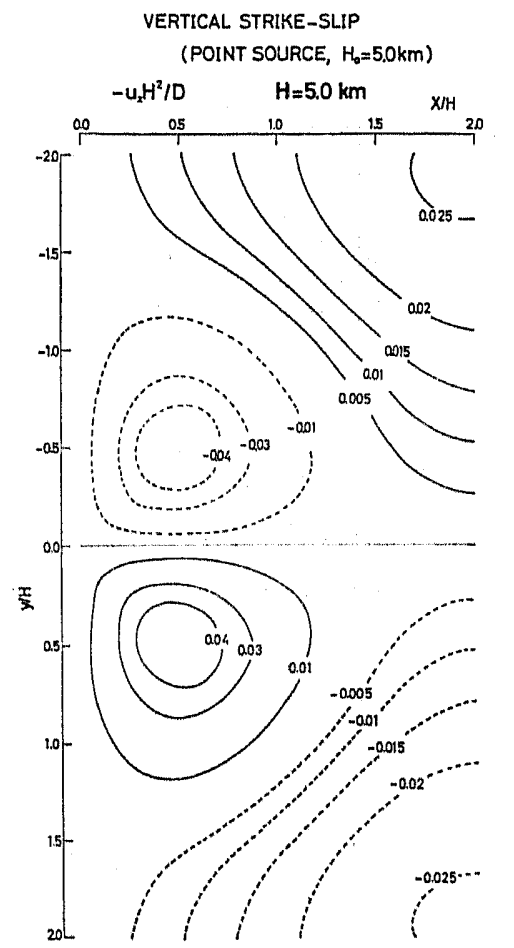

(a)

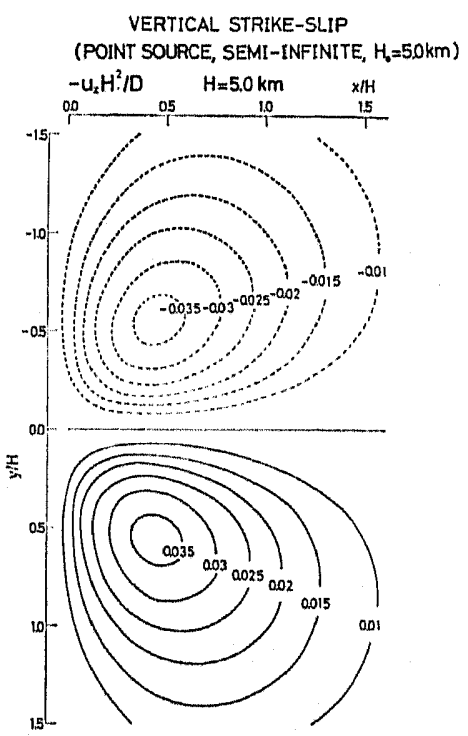

(b)

Fig. 10. Contour map of vertical displacements at the surface due to the vertical strike-slip fault (point source case).

(a) model I.

(b) model II.
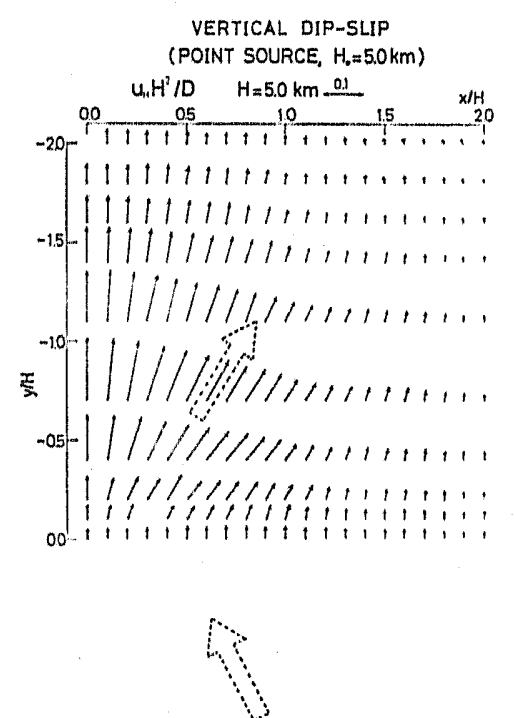

(a)
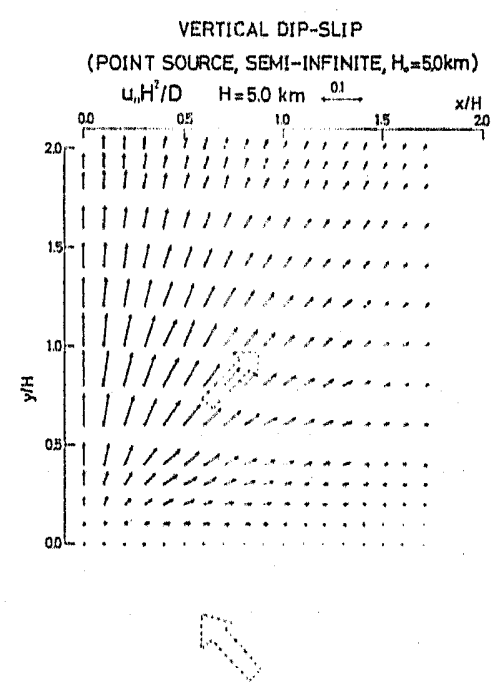

(b)

Fig. 11. Vector map of horizontal displacements at the surface due to the vertical dipslip fault (point source case).

(a) model $\mathrm{I}$.

(b) model II. 


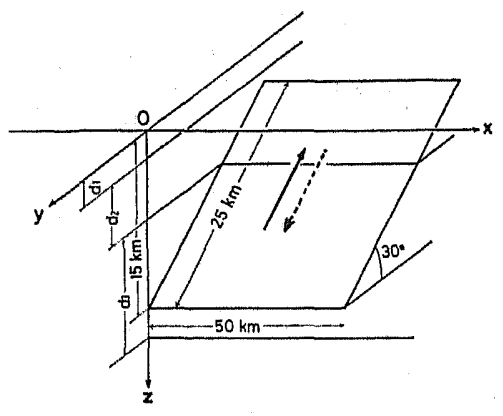

DISPLACEMENT VERTICAL COMPONENT model I

FOCAL DEPTH $=15.0 \mathrm{~km}$ UNIT LENGTH $=25.0 \mathrm{~km}$

DIP ANGLE $=30^{\circ}$, SLIP ANGLE $=90^{\circ}$

$w=25.0 \mathrm{~km}, L=50.0 \mathrm{~km}$

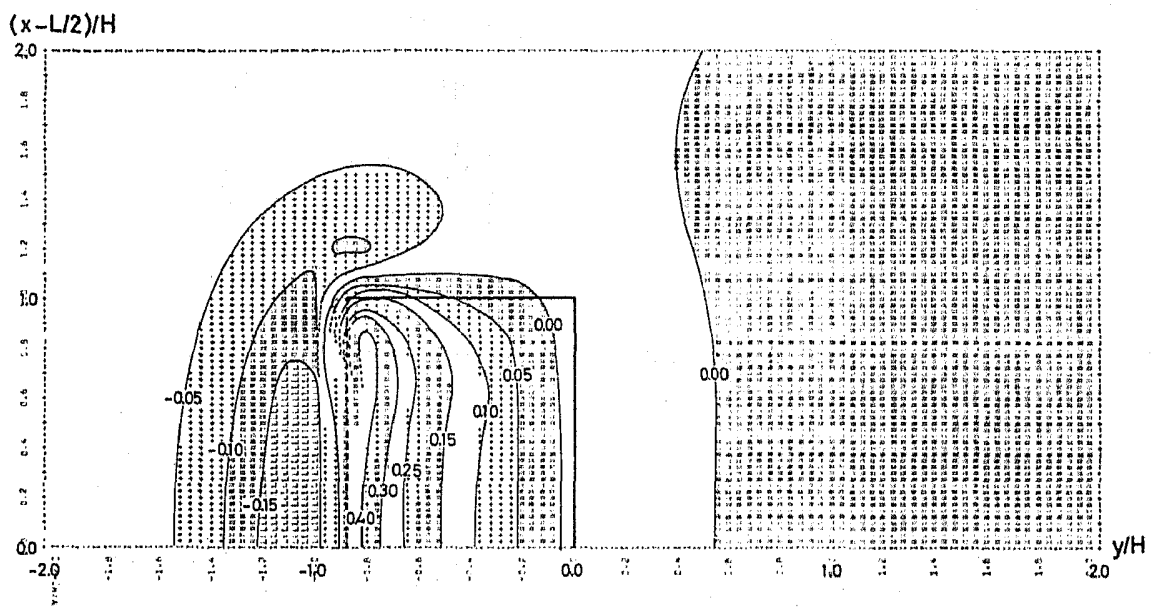

(a)

Fig. 12. Vertical displacements due (a) model I. Geometry is given on the right side.

for the two models, which will be shown in the next paper.

Figure 11 illustrates comparison of horizontal components for the vertical dip-slip fault. Main features are not so different but it should be pointed out the following two points: Magnitudes of deformation for model $I$ are, as a whole, greater than that for model II, and the horizontal displacements for model I appear even along the strike direction of the fault ( $x$-axis), while no horizontal displacement does for model II.

In drawing of Figs. 9, 10 and 11, machine program was not used. Figs. 5 and 6 are examples of outputs of Program IV-A and IV-B, respectively. 
DISPLACEMENT VERTICAL COMPONENT model II

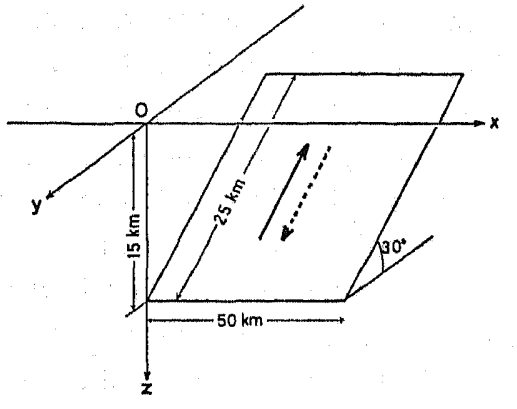

FOCAL DEPTH $=15.0 \mathrm{~km}$ UNIT LENGTH $=25.0 \mathrm{~km}$

DIP ANGLE $=30^{\circ}$, SLIP ANGLE $=90^{\circ}$ $W=25.0 \mathrm{~km}, L=50.0 \mathrm{~km}$

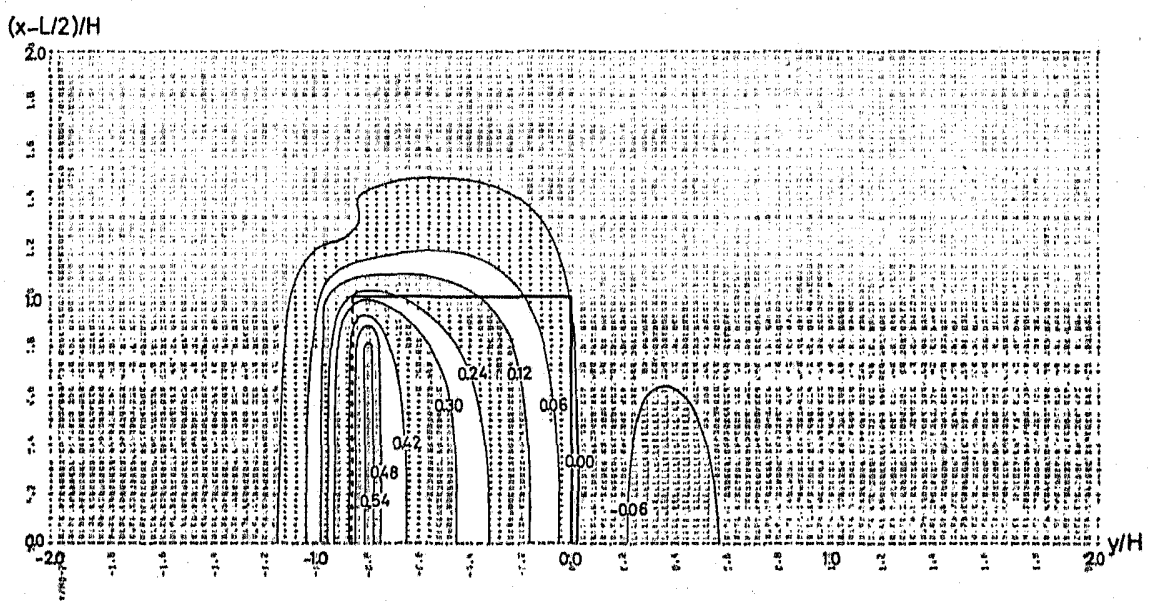

(b)

to the dip-slip fault with dimension.

(b) model II. Geometry is given on the right side.

iii) The case of the fault with dimensions (Fig. 12)

For reverse dip-slip fault with dip-angle, $30^{\circ}$, contour of vertical displacements $\left(-u_{z} / D\right)$ are shown in Fig. 12. For model I (Fig. 12(a)), the fault spreads over the upper two layers. Since unit of length, $H$, is taken as $25 \mathrm{~km}$, these contour maps cover the area $x=25.0 \mathrm{~km}$ ( $=$ fault length $/ 2) \sim 75.0 \mathrm{~km}$ and $y=-50.0 \mathrm{~km} \sim 50 . \mathrm{km}$.

In this example, dislocation over the fault plane is simply taken as constant. If the seismic moment is considered to be constant over the fault plane, then the dislocation shoud be taken as one inversely proportional to rigidity 
in each layer. In the present program, the information about seismic moment is given by the input data, MOM (see the flow chart of Program III).

In this computation, distance between two neighbouring points at the surface where displacements are calculated is taken as $0.2 \times$ (unit length), and then by applying interpolation formula, the contour map is drawn by taking 0.1 mesh point interval. For the case when one corner of the fault is quite close to the surface, and distribution of dislocation over the fault plane is not uniform, smaller interval may be required in order to follow the fine feature of the displacement field. But taking many other effects on the very near field observations and their accuracies in to account, it seems unnecessary to calculate with much denser mesh points.

\section{Summary}

Crustal deformation when the fault spreads over several layers in a multilayered medium is investigated.

Main purpose of the present study is to establish the machine program system. In this program, designating the crustal structure (layer thicknesses, dilational and shear velocities and densities), fault length, fault width, focal depth (origin of the fault), dip angle and slip angle, surface deformation is calculated and plotted on the vector or contour map if necessary. The area to be computed is controlled by giving unit of length.

Several numerical examples are presented to compare surface deformation in a layered medium (model $I$ in Table 3 ) with that in a semi-infinite medium (model II).

Surface deformation must depend on structure, fault type and fault dimension and, in order to obtain general conclusions on the difference between two models, it should be necessary to compute and compare for various structures and fault models. However, as far as the present models are concerned, we can point out the followings:

(1) The maximum deformation for model I is generally greater than that for model II, for both the dip-slip and the strike-slip fault considered here.

(2) Pattern of the horizontal component seems to be not quite different for two models as a whole, but details are different. For example, the vertical dip-slip fault does not yield the horizontal displacement along the strike direction of the fault for model II, but does for model I.

(3) For the vertical component, it is observed the reverse area for model I where the direction of deformation is opposite to that for model II. Hence, patterns for two models are similar only in the immediate vicinity of the area where the fault is close to the surface and are different outside of this area.

Tilt and strains on the surface due to the present model will be shown in 
the next paper.

The computations involved were made partly on a HITAC 8800,8700 at the Computer Center, University of Tokyo, and partly on an IBM SYSTEM 360 MODEL 195 through the UNICON.

\section{REFERENCES}

ANDo, M., A fault-origin model of the great Kanto earthquake of 1923 as deduced from geodetic data, Bull. Earthq. Res. Inst., 49, 19-32, 1971.

Ben-Menahem, A. and A. Grllon, Crustal deformation by earthquakes and explosions, Bull. Seism. Soc. Amer., 60, 193-215, 1970.

Ben-Menahem, A., S.J. Sighn, and F. Solomon, Static deformation of a spherical earth model by internal dislocations, Bull. Seism. Soc. Amer., 59, 355-372, 1961.

BurRidge, R. and L. KNopoff, Body force equivalents for seismic dislocations, Bull. Seism. Soc. Amer., 54, 1875-1888, 1964.

CANITEZ, N. and M.N. TokzOZz, Static and dynamic study of earthquake source mechanism; San Fernando Earthquake, J. Geophs. Res., 77, 2583-2594, 1972.

Chinnery, M.A., The deformation of ground around surface faults, Bull. Seism. Soc. Amer. 51, 355-372, 1961.

FrTcH, T.J. and C.H. ScHolz, Mechanism of underthrusting in southwest Japan; A model of convergent plate interaction, J. Geophs. Res., 76, 7260-7292, 1971.

HASTIE, L.M. and J.C. SAVAGE, A dislocation model for the 1964 Alaska earthquake, Bull. Seism. Soc. Amer., 60, 1389-1392, 1970.

Mansinha, L. and D.E. Smylie, The displacement field of inclined faults, Bull. Seism. Soc. Amer., 61, 1433-1440, 1971.

MARUYAma, T., Statistical elastic dislocations in an infinite and semi-infinite medium, Bull. Earthq. Res. Inst., 42, 289-368, 1964.

PRESs, F., Displacements, strains, and tilts at tele-seismic distances, J. Geophs. Res., 70, 23952412, 1965 .

SATo, R., Formulations of Solutions for earthquake source models and some related problems, J. Phys. Earth, 17, 101-110, 1969.

SAto, R., Crustal deformation due to dislocation in a multi-layered medium, J. Phys. Earth., 19, 31-46, 1971.

SAYAGE, J.C. and L.M. HASTre, Surface deformation associated with dip-slip faulting, $J$. Geophys. Res., 71, 4897-4904, 1966.

SAVAGE, J.C. and L.M. HAStre, A dislocation model for the Fairview Peak, Nevada, earthquake, Bull. Seism. Soc. Amer., 59, 1937-1948, 1969.

SCABORougr, J.B., Numerical mathematical analysis, pp. 516-557, The Johns Hopkins Press, Baltimore, 1962.

STEKetee, J.A., On Volterra's dislocations in a semi-infinite elastic medium, Can. J. Phys., 36, 192-205, 1958.

Steketee, J.A., Some geophysical applications of the elasticity theory of dislocations, Can. J. Phys., 36, 1168-1198, 1958.

WaLsh, J.B., Dip angle of faults as calculated from surface deformation, $J$. Geophys. Res., 74, 2070-2080, 1969. 